\title{
Ideas y opiniones del educador de museos en relación a las Tecnologías de la Información y la Comunicación ${ }^{1}$
}

\section{Ideas and opinions of museums educator about the Information and Communication Technology}

\author{
José Miguel CORREA, Daniel LOSADA y Estibaliz JIMENEZ DE ABERASTURI \\ Universidad del País Vasco
}

Recibido: Marzo 2012

Aceptado: Abril 2012

\begin{abstract}
Resumen
Analizamos en este artículo las ideas y opiniones de los educadores de museos sobre las ventajas e inconvenientes de la integración y uso de las Tecnologías de la Información y de la Comunicación en los museos y espacios de presentación del patrimonio. Para el desarrollo de la investigación, en la que han participado 45 educadores de museos, se ha utilizado la metodología por encuesta, analizando los aspectos a tener en cuenta en su integración y utilización, así como las ventajas e inconvenientes que aportan en la realidad de los museos. Los resultados obtenidos muestran opiniones favorables a la integración y uso de las mismas en estos contextos de aprendizaje y una actitud positiva hacia las tecnologías por parte de los educadores de museos.
\end{abstract}

Palabras clave: museos, educadores, TIC, opiniones, creencias

\begin{abstract}
We analyze in this article the views and opinions of museum educators about the advantages and disadvantages of integration and use of Information Technology and Communication in the museums and heritage presentation. For the development of research, which involved 45 educators from museums, we have used the survey methodology, analyzing the aspects to consider in their integration and use, and the advantages and disadvantages they bring to reality museums. The results show favorable opinions on the integration and use of them in these contexts of learning and a positive attitude towards technology by museum educators.
\end{abstract}

Keywords: museums, educators, ict, opinions, beliefs

\footnotetext{
${ }^{1}$ Proyecto de investigación: Innovación educativa con tecnologías digitales: cambio tecnológico y transformaciones culturales y educativas. (Gobierno Vasco IT 433-10)
} 
La incorporación de los nuevos medios digitales en los museos y espacios de presentación del patrimonio ha ido en paralelo a la extensión y difusión de estos recursos en todas las esferas sociales. Como centros de cultura, ocio y atracción turística, los museos afortunadamente han sabido transformarse y ser capaces de convertirse en centros de experimentación, innovación e investigación de lo que ha venido siendo las últimas apariciones tecnológicas.

De esta manera los museos y espacios de presentación del patrimonio han tenido la oportunidad de compaginar el reto de la alfabetización científica y cultural con el de la alfabetización digital. Consagrados investigadores de la educación en museos supieron vaticinar las excelentes oportunidades que los nuevos medios digitales abría a estas instituciones y el impacto previsible en la transformación de sus objetivos y actividades didácticas (Falk y Dierking, 2000; Hein, 1998; Hooper-GreenHill, 1999).

Durante los últimos quince años, gracias a políticas concretas de integración de la tecnología digital en los museos, se ha multiplicado la presencia y aplicaciones tecnológicas en estos centros, transformándose las tareas habituales de gestión patrimonial, investigación y difusión de sus contenidos científicos y culturales.

Una tradición tecnológica que ha evolucionado desde los tradicionales recursos audiovisuales del siglo pasado a los actuales recursos tecnológicos digitales, diversificándose sus recursos desde las páginas web hasta la realidad aumentada, pasando por la edición de contenidos digitales y variadas formas de participación ciudadana. Todo ello ha hecho de los dispositivos y recursos tecnológicos que estas instituciones ponen al alcance de los visitantes, un atractivo más y un factor de competitividad y prestigio institucional, de manera que los visitantes esperan estas visitas a centros de ciencia o museos de cualquier temática, como ocasiones ideales para experimentar con dispositivos tecnológicos de uso cotidiano, transformando la experiencia propia de la visita al museo.

\section{Museos, nuevos medios y aprendizaje}

La transformación tecnológica, afecta desde hace años a la manera de vivir los museos. No solo a cómo conseguimos nuestras entradas sino también a la manera de gestionar nuestras visitas y enriquecernos de los contenidos digitales e informaciones que el museo pone a disposición del visitante. Todo ello ha afectado especialmente a todas las funciones del museo, despertando nuevos retos a los educadores e investigadores educativos en estos contextos de aprendizaje. Si en una primera fase de transformación del contexto museístico fue la virtualidad de los visitantes, una de las claves, actualmente no solo se ha mantenido el atractivo de estas visitas en la web sino que se ha potenciado el interés de las visitas presenciales, impulsado por un conjunto de aplicaciones para dispositivos móviles que han transformado la experiencia museística.

Esta transformación de la experiencia ha traído consigo la emergencia de un nuevo rol mucho más activo de los visitantes de lo que tradicionalmente era, gracias no solo a la conectividad de la que se dispone y al acceso a una multiplicidad de recursos informativos con los nuevos dispositivos móviles, sino también al aumento del nivel de 
alfabetización digital de los ciudadanos, que permite y promueve nuevas maneras de interpretar el patrimonio. Esta actitud es muy beneficiosa para los visitantes y para la propia institución pues promueve nuevas maneras de ver, de interpretar, de comprender y de participar en nuestras visitas y con los museos. Lo más positivo es la aparición de un rol creativo en el visitante que gracias a los recursos tecnológicos de que dispone, desea reinterpretar, crear y dejar huella en sus visitas, rompiendo ciertas barreras tradicionales de observación pasiva y de no participación que se han levantado entre la cultura y la ciencia y el visitante de los museos.

A la vez, la disponibilidad de recursos tecnológicos ha permitido la transformación de estos contextos, mediante aplicaciones concretas como los "mobile apps", "tablets" o la "realidad virtual". Estas tecnologías permiten a los visitantes la personalización de los itinerarios y la ruptura con el clásico paradigma de enseñanza y aprendizaje en los museos y espacios de presentación del patrimonio como exposición a los objetos y la información, más que generar oportunidades de experimentación para el aprendizaje.

Todo esto unido a las nuevas expectativas sociales sobre participación cultural, está transformando las experiencias educativas de los museos convirtiéndose en un proceso más dialógico, activo, interpretativo a la vez que subjetivo y sobre todo emocional. Una experiencia que ha pasado de ser contemplativa a convertirse en un pretexto para dejar huella. Si algo han aportado estos recursos tecnológicos, en relación con la adquisición del conocimiento y el aprendizaje, ha sido la posibilidad de crear comunidades de práctica y compartir socialmente el conocimiento y representarlo en múltiples formatos y lenguajes.

\section{Museos, tecnología y aprendizaje}

Durante estos últimos años hemos venido desarrollando diferentes investigaciones sobre la aplicación de la tecnología en museos y espacios de presentación del patrimonio (Correa, 2011; Correa y Jimenez de Aberasturi, 2011; Ibañez, Correa et al, 2011; Ibañez, Asensio, Correa, 2011; Correa, Leon, y Fernandez, 2010; Martinez y Correa, 2009; Correa, 2008; Correa, Ibañez y Jimenez de Aberasturi, 2006; Correa e Ibañez, 2005) De esta manera hemos analizado el impacto de la aparición de internet y los museos virtuales en la difusión de la ciencia, la aplicación de dispositivos móviles (smartphones, PDas, Gps) o el fenómeno de la participación ciudadana en los museos favorecida por la aparición de las tecnologías sociales.

Conforme hemos ido analizando la presencia y usos de las tecnologías digitales en los museos y espacios de presentación del patrimonio nos hemos dado cuenta por una parte, de la rapidez de aplicación y transferencia al museo de estas innovaciones tecnológicas; por otra parte, hemos sido conscientes de la fertilidad investigadora en esta temática y el aumento exponencial de publicaciones sobre la misma. Numerosos proyectos asociados a los más importantes museos nacionales e internacionales demuestra el progresivo incremento y la necesidad de incorporar estas tecnologías como una estrategia de marketing, innovación y demanda de los usuarios. 
De esta manera encontramos tanto a nivel internacional museos de gran prestigio como el Moma (http://www.moma.org/), Exploratorium de San Francisco (http://www.exploratorium.edu/), Museo Británico (http://www.britishmuseum.org), liderando iniciativas orientadas a acercar las tecnologías más novedosas al visitante de los museos. También se han dado múltiples iniciativas de destacados museos a nivel nacional Thysen (http://www.museothyssen.org/en/thyssen/comunidad) o el CosmoCaixa Barcelona, incluso podríamos identificar muchos proyectos museísticos de integración de la tecnología a niveles locales en las diferentes comunidades autónomas (ver por ejemplo: http://www.gipuzkoamuseobirtuala.net/index2.php?id_lingua=2).

Esta presencia tecnológica cada vez mayor está teniendo una influencia decisiva en la transformación de las actividades educativas de los museos.

\section{Los educadores de museos y la tecnología}

Como en otras actividades profesionales, las tecnologías digitales también han influido en el rol de los educadores de museos. La presencia tecnológica ha supuesto nuevas tareas y otras maneras de comprender y gestionar las funciones educativas de los museos. No solo se demanda niveles de competencia digital, sino también conocimiento de aplicaciones educativas, experiencias y utilización recursos digitales para poner en práctica los diversos programas educativos en museos y espacios de presentación del patrimonio.

Como en el ámbito escolar las investigaciones sobre el impacto de las TIC en la educación han demostrado, y pensamos que ello también es extensible al ámbito museístico, la importancia de las actitudes y creencias de los educadores hacia las tecnologías. Siendo un factor de primer orden en su utilización y rendimiento Otros factores endógenos identificados como decisivos en la utilización de los recursos tecnológicos, aportados por las investigaciones son: la competencia TIC, la actitud positiva, la colaboración con los compañeros, la visión institucional de las TIC así como el clima de trabajo y la disponibilidad de infraestrucuras y tiempo (Ely, 1999; Mumtaz, 2000; Drent y Meelissen, 2008; BECTA 2009), Balanskat, Blamire y Kefala, 2006; Losada, Karrera y Jimenez de Aberasturi, 2012).

Un contexto tecnológico cada vez más atractivo pero exigente condiciona las competencias tecnológicas de los educadores de los museos. Pero ¿cuáles son estas opiniones?, ¿cuáles son las ventajas que creen que les aportan?, ¿cuáles son desde su punto de vista los elementos importantes a tener en cuenta para una integración y uso eficiente de las TIC en los museos? y ¿Qué dificultades perciben en la integración y utilización de las TIC en los museos?

En base a estas cuestiones, el presente estudio se propone el siguiente objetivo:

- Identificar las ideas, opiniones y visión de las Tecnologías de la Información y la Comunicación de los educadores de museos en su centro de trabajo. 


\section{Método}

Con el objeto de recopilar la perspectiva de los educadores de museos sobre las Tecnologías de la Información y la Comunicación en su entorno laboral, se ha utilizado la metodología por encuesta. Este procedimiento es uno de los más comunes de recogida de información dentro de la investigación en ciencias sociales, puesto que es idóneo para obtener datos sobre fenómenos que no pueden ser observados directamente (Colás y Buendia, 1994). En este caso en particular, la necesidad de recopilar las ideas, opiniones y visión de las Tecnologías de la Información en los museos obliga a llevar a cabo este tipo de metodología para un acercamiento fidedigno a la realidad a investigar.

\section{Participantes}

En este estudio han participado 45 educadores de museos de la Comunidad Autónoma de Cataluña en activo. Estos participantes asistieron al taller titulado "Tecnologías para la creatividad" dentro de las "XIV Jornadas de Museus i Educació: Museus i actvitats on line" celabradas en el Museum Maritim de Barcelona el 24 y 25 de marzo del 2011. Esta muestra incidental está compuesta por 36 mujeres y 6 hombres, con una edad repartida uniformemente. El $64,4 \%$ de los encuestados tienen menos de 40 años, mientras que el restante $36,6 \%$ tiene más de 40 años. Por último, la totalidad de los educadores tienen una experiencia profesional en centros museísticos igual o superior a los 5 años de desempeño.

\section{Procedimiento}

Los participantes en cumplimentaron un cuestionario realizado ad hoc para recoger las opiniones, visiones y actitudes sobre la TIC del colectivo de educadores de museos. Los datos recogidos fueron analizados mediante el paquete estadístico SPSS en su versión 19 para MacOS.

En primer lugar, se han analizado las garantías estadísticas del cuestionario mediante un análisis de la fiabilidad de la escala. Se ha aplicado la prueba Alpha de Cronbach. Este estadístico es uno de los coeficientes más utilizados a la hora de determinar si una escala es fiable y por lo tanto, si dispone de consistencia interna en base a una correlación positiva entre sus ítems que garanticen que la medición en cierto grado forma una entidad en común en relación al objeto a analizar.

En segundo lugar, los datos recogidos en el cuestionario se han utilizado para identificar la posición ante la tecnología de los educadores de museos mediante el análisis descriptivo de los ítems que componen el cuestionario. En base a esto, se han realizado para cada ítem el cálculo de frecuencias, porcentajes, promedio y desviación típica. 


\section{Instrumentos de Medida}

A partir de varias etapas, se ha diseñado el "Cuestionario sobre las Concepciones de las TIC en el Ámbito Museístico" para la recogida de datos en este estudio. Con este instrumento se busca delimitar dentro de la identidad del educador de museos la importancia de las TIC y su papel transformador en su labor profesional.

En una primera etapa, aparte de ciertos datos generales relacionados con el encuestado (edad, sexo y años de experiencia), se propusieron inicialmente 42 ítems para su respuesta. Con el objeto de fortalecer la validez de contenido de los mismo, se sometieron a criterio de tres jueces. Las sugerencias recibidas modificaron la formulación de varias preguntas y provocaron reducir a 40 ítems el cuestionario. Aunque no existe la intención de crear dimensiones dentro de la escala, los ítems giran en torno a las siguientes temáticas:

- Elementos importantes a tener en cuenta para una integración y uso eficiente de las TIC en los museos (15 ítems)

- Dificultades en la integración y utilización de las TIC en los museos (10 ítems)

- Ventajas que aportan las TIC en la realidad de los museos (15 ítems)

Estas preguntas están construidas mediante una escala valorativa de tipo Likert de cinco alternativas. Los encuestados que han respondido a cada uno de los ítems deben indicar con valor o dirección su grado de acuerdo con los diferentes afirmaciones recogidas, oscilando entre uno (Totalmente en desacuerdo) y cinco (Totalmente de acuerdo).

En una segunda etapa, se ha llevado a cabo un análisis de la fiabilidad de la estructura dimensional. El resultado del alfa de Cronbach ha sido muy bueno, con un puntaje de 0,842 puntos. Asimismo, la retirada de ninguno de los ítems de la escala mejoraba significativamente este valor.

\section{Resultados}

Las evidencias recogidas en este estudio han permitido realizar un análisis de los resultados en base a tres grandes temáticas:

\section{Ventajas que aportan las TIC en la realidad de los museos}

Los datos analizados informan de una visión positiva y muy generalizada entre los Educadores de Museos ante las ventajas que ofrece la integración y utilización de las Tecnologías de la Información y la Comunicación en su lugar de trabajo. Aunque no en todas las preguntas la mitad de lo encuestados se posicionan de acuerdo o totalmente de acuerdo con las ventajas planteadas en este cuestionario (Tabla 1), todos lo ítems superan la media de la escala (2,5 puntos). 


\begin{tabular}{|l|c|c|c|}
\hline \multicolumn{1}{|c|}{ Ítem } & Media & DT & $\begin{array}{c}\text { \% de encuestados de } \\
\text { acuerdo y totalmente } \\
\text { de acuerdo }\end{array}$ \\
\hline $\begin{array}{l}\text { Las TIC nos ayudan en la difusión de materiales } \\
\text { educativos de nuestros museos }\end{array}$ & 4,22 &, 560 & 93,3 \\
\hline $\begin{array}{l}\text { Las Tic transforman la imagen y visibilidad del } \\
\text { museo }\end{array}$ & 4,22 &, 560 & 82,2 \\
\hline $\begin{array}{l}\text { Las TIC deben de ayudarnos a diversificar los } \\
\text { programas educativos de los museos }\end{array}$ & 4,11 &, 804 & 77,8 \\
\hline $\begin{array}{l}\text { Las TIC transforman los sistemas de acceso a } \\
\text { las colecciones y exposiciones de los museos }\end{array}$ & 3,96 &, 852 & 76,6 \\
\hline $\begin{array}{l}\text { Las TIC transforman la planificación, gestión y/o } \\
\text { organización del museo }\end{array}$ & 3,80 &, 968 & 68,9 \\
\hline $\begin{array}{l}\text { Las TIC mejoran los materiales educativos de los } \\
\text { museos }\end{array}$ & 3,80 &, 842 & 66,7 \\
\hline $\begin{array}{l}\text { Las TIC mejoran la formación permanente de los } \\
\text { educadores de los museos }\end{array}$ & 3,64 &, 830 & 64,4 \\
\hline $\begin{array}{l}\text { Las TIC nos ayudan a transformar nuestras pro- } \\
\text { puestas educativas haciéndolas más activas }\end{array}$ & 3,71 &, 757 & 63,2 \\
\hline $\begin{array}{l}\text { Las TIC transforman las metodologías educati- } \\
\text { vas de los museos }\end{array}$ & 3,64 &, 802 & 62,2 \\
\hline $\begin{array}{l}\text { Las TIC nos permiten mejorar el tratamiento de } \\
\text { personas con necesidades educativas especiales }\end{array}$ & 3,60 &, 963 & 62,3 \\
\hline $\begin{array}{l}\text { Gracias a las TIC los programas educativos del } \\
\text { museo son más atractivos }\end{array}$ & 3,36 &, 981 & 44,4 \\
\hline $\begin{array}{l}\text { Las TIC consiguen fomentar el trabajo colabora- } \\
\text { tivo entre los educadores de los museos }\end{array}$ & 3,42 &, 812 & 53,3 \\
\hline $\begin{array}{l}\text { Los lenguajes audiovisuales junto con Internet } \\
\text { mejoran la calidad educativa de los programas de } \\
\text { los museos }\end{array}$ & 3,49 &, 815 & 46,7 \\
\hline $\begin{array}{l}\text { Las redes sociales nos ayudarán a cambiar la } \\
\text { metodología educativa de los museos }\end{array}$ & 3,16 &, 796 & 31,1 \\
\hline $\begin{array}{l}\text { Gracias a las TIC los visitantes en los museos } \\
\text { están más motivados }\end{array}$ & 3,09 &, 793 & \\
\hline Tabl. Meda dovion to & & \\
\hline
\end{tabular}

Tabla 1. Media, desviación típica y porcentaje de encuestados de acuerdo y totalmente de acuerdo en la valoración de los ítems del cuestionario relacionados con las ventajas de la integración de las TIC en los museos.

En este sentido, existe un consenso muy amplio entre los educadores a la hora de ver las herramientas tecnológicas como transformadoras de la realidad del museo y de la visión que ofrece de cara al exterior. En el terreno de la imagen del museo, el 82,2\% de los encuestados están de acuerdo o totalmente de acuerdo que mejoran la visibilidad del museo. 
Profundizando en el impacto en el museo, el 76,6\% de los educadores reconocen la posibilidad de transformar los sistemas de acceso a las colecciones y a las exposiciones. Asimismo, estas herramientas son consideradas por más del $65 \%$ de los encuestados como una buena oportunidad para el ámbito museístico en relación a los materiales y los programas educativos, así como para transformar la planificación, la gestión y la organización de su ámbito de trabajo.

Las ventajas inherentes de las TIC a la hora de facilitar la difusión $(93,3 \%)$ y diversificación $(77,8 \%)$ de materiales y programas educativos, así como la posibilidad de dar respuesta a las personas con necesidad educativas especiales $(62,3 \%)$ han quedado de manifiesto, incluso llegando a considerarlas por tres de cada cinco encuestados como transformadoras de las metodologías educativas de los museos. En esta línea, más del $60 \%$ de los encuestados considera que las TIC les ayudan a realizar propuestas educativas mucho más activas y mejoran su formación permanente.

Sin embargo, entre los encuestados existe también cierto grado de indecisión sobre la capacidad transformadora de los TIC para mejorar los programas educativos de los museos en diversos aspectos. Más del $40 \%$ de los encuestados no está ni de acuerdo ni en desacuerdo con su potencial motivador, atrayente ni de mejora de la calidad de los materiales, dudando del papel beneficioso de Internet y de las redes sociales en los museos.

\section{Elementos importantes a tener en cuenta para una integración y uso eficiente de las TIC en los museos}

En este segundo conjunto de ítems se recogen aspectos nucleares necesarios para un impacto efectivo de las herramientas tecnológicas en el ámbito museístico. Los resultados obtenidos (Tabla 2) informan que todas las afirmaciones recogidas son importantes a la hora de implementar lo tecnológico en los museos, debido a que la valoración realizada en todos los ítems informan de valores por encima de la media del cuestionario (2,5 puntos). Asimismo, la mayoría de los educadores encuestados (entre el 68,9\% y el $95,5 \%)$ están de acuerdo o totalmente de acuerdo con los enunciados planteados. En este sentido, la idoneidad del ambiente de trabajo, la necesidad de trabajar en equipo y las condiciones del entorno de trabajo que fomenten la innovación son elementos valorados muy positivamente.

En relación al ambiente de trabajo, la importancia atribuida a las TIC para su uso en el ámbito museístico es considerada como definitoria para una gran mayoría de los educadores de museos $(73,3 \%)$. Sin embargo, el elemento con un mayor consenso entre los encuestados $(95,5 \%)$ se sitúa en la necesidad de una actitud positiva ante las TIC de todas las personas relacionadas con el museo, incluyendo educadores, directivos y visitantes en general.

Además, para tres de cada cuatro encuestados se ha de generar un ambiente de trabajo estimulante. En esta línea, el estimulo de la participación de los educadores de museos en proyectos de innovación TIC, incentivar el uso TIC en programas y actividades educativas, así como dotar de reconocimiento institucional y/o profesional son elementos también importantes a considerar para más de un tercio de la muestra recogida. 


\begin{tabular}{|c|c|c|c|}
\hline Ítem & Media & DT & $\begin{array}{l}\text { \% de encuestados de } \\
\text { acuerdo y totalmente } \\
\text { de acuerdo }\end{array}$ \\
\hline $\begin{array}{l}\text { Para la utilización de las TIC es necesaria la actitud } \\
\text { positiva de los educadores, directivos del museo y } \\
\text { visitantes en general }\end{array}$ & 4,69 & ,557 & 95,5 \\
\hline $\begin{array}{l}\text { Las TIC hay que integrarlas de forma cooperativa, } \\
\text { crítica y creativa en los programas educativos de los } \\
\text { museos }\end{array}$ & 4,47 & ,588 & 95,5 \\
\hline $\begin{array}{l}\text { Una gran preparación y habilidades en TIC de los } \\
\text { responsables de la innovación en los museos favo- } \\
\text { rece su utilización }\end{array}$ & 4,44 & 659 & 91,1 \\
\hline $\begin{array}{l}\text { Tenemos que fomentar la formación permanente de } \\
\text { los educadores en TIC }\end{array}$ & 4,33 & ,707 & 91,1 \\
\hline $\begin{array}{l}\text { Es necesaria una buena coordinación y trabajo en } \\
\text { equipo de los educadores de museos para integrar } \\
\text { las TIC en sus actividades }\end{array}$ & 4,29 & ,661 & 88,9 \\
\hline $\begin{array}{l}\text { El museo debe de utilizar las TIC para la creación } \\
\text { de redes de colaboración externas, redes de investi- } \\
\text { gación, redes de educadores de museos }\end{array}$ & 4,27 & ,780 & 88,9 \\
\hline $\begin{array}{l}\text { Hay que estimular la participación de los educado- } \\
\text { res de los museos en proyectos de innovación TIC }\end{array}$ & 4,22 & 670 & 86,7 \\
\hline $\begin{array}{l}\text { Se necesita disponibilidad de espacios y recursos } \\
\text { informáticos para el desarrollo de innovaciones con } \\
\text { TIC }\end{array}$ & 4,31 & ,973 & 84,5 \\
\hline $\begin{array}{l}\text { Para los educadores de los museos el reconocimien- } \\
\text { to institucional y/o profesional de la innovación con } \\
\text { TIC es importante }\end{array}$ & 4,27 & ,720 & 84,4 \\
\hline $\begin{array}{l}\text { El compromiso e iniciativa por parte de los educa- } \\
\text { dores de los museos determina la integración de las } \\
\text { TIC en los programas educativos de los museos }\end{array}$ & 4,07 & ,939 & 80,0 \\
\hline $\begin{array}{l}\text { En los museos tenemos que generar un ambiente de } \\
\text { trabajo estimulante para la innovación con TIC }\end{array}$ & 4,09 & ,996 & 75,5 \\
\hline $\begin{array}{l}\text { Hay que incentivar el uso de TIC en los programas } \\
\text { y actividades educativas de los museo }\end{array}$ & 4,02 & 839 & 75,5 \\
\hline $\begin{array}{l}\text { La importancia atribuida a las TIC es fundamental } \\
\text { para su integración }\end{array}$ & 3,89 & 910 & 73,3 \\
\hline $\begin{array}{l}\text { Tenemos que utilizar las TIC para la ampliación de } \\
\text { la oferta formativa y/o creación de nuevos entornos } \\
\text { de enseñanza aprendizaje dirigidas al público }\end{array}$ & 3,93 & ,751 & 73,3 \\
\hline $\begin{array}{l}\text { Tenemos que fomentar la creación de grupos de } \\
\text { trabajo entre educadores de museos para el uso de } \\
\text { las TIC }\end{array}$ & 3,98 & 1,03 & 68,9 \\
\hline
\end{tabular}

Tabla 2. Media, desviación típica y porcentaje de encuestados de acuerdo y totalmente de acuerdo en la valoración de los ítems del cuestionario relacionados con los elementos a tener en cuenta en la integración y uso de las TIC en los museos. 
En relación al trabajo en equipo, casi la totalidad de los encuestados (95,5\%) está de acuerdo o totalmente de acuerdo que el modo de integración de las TIC en los programas educativos de los museos debe darse de un modo cooperativo, crítico y creativo. Desde esta perspectiva, el compromiso e iniciativa de los educadores de museos es considerada como fundamental por cuatro de cada cinco encuestados.

Es por ello que la mayoría de la muestra propone fomentar la creación grupos de trabajo entre los educadores de museos $(68,9 \%)$ y considera necesaria una buena coordinación y trabajo en equipo para integrar las TIC en la vida diaria de los museos $(88,9 \%)$. Una medida que apoyan mayoritariamente $(88,9 \%)$ supone utilizar las TIC para la creación de redes de colaboración externa, redes de investigación o simplemente de intercambio entre educadores de museos.

En relación a las condiciones del entorno de trabajo que fomentan la innovación, el $73,3 \%$ de los encuestados postulan por ampliar la oferta formativa y crear nuevos entornos de enseñanza-aprendizaje dirigidas al publico. A juicio de la mayoría de la muestra, la disponibilidad de espacios y recursos informáticos para el desarrollo de innovaciones $(84,5 \%)$, el fomento de la formación permanente de los educadores de museos $(91,1 \%)$ y una gran preparación en habilidades TIC de los responsables de innovación de los museos $(91,1 \%)$ favorecen este y otros usos.

\section{Dificultades en la integración y utilización de las TIC en los museos}

A través del análisis de los datos reportados mediante la valoración de las afirmaciones (Tabla 3), se aprecia tres grandes bloques de dificultades en relación a la implementación y uso de las TIC en el ámbito museístico.

En primer lugar, los educadores de museos han quedado evidenciados como foco de obstáculos a la hora de integrar las TIC en la práctica habitual. Más de la mitad de los encuestados opinan que la falta de competencias digitales de este colectivo dificulta la innovación de los programas educativos con TIC. Asimismo, existe una falta de consenso sobre si existe claramente un respaldo de la innovación con TIC por parte de estos profesionales puesto que casi la mitad de la muestra ni está de acuerdo ni en desacuerdo con esta cuestión.

Sin embargo, la mayoría de los encuestados no cree que entre las dificultades resida la desconfianza hacia las TIC por parte de los educadores de museos $(75,6 \%)$ o una falta de valoración positiva en el terreno instructivo de las TIC en los programas educativos $(80 \%)$. Sólo uno de cada cuatro valora estos impedimentos como nucleares.

En segundo lugar, las características de los visitantes a los museos pueden ser otra fuente de dificultades. No obstante, existe cierto grado de indecisión entre los encuestados a la hora de situar la actitud negativa de los mismos, así como su falta de conocimientos y habilidades básicas TIC a la hora de limitar las TIC en el ámbito museístico. Más del $40 \%$ de los encuestados no esta ni de acuerdo ni en desacuerdo con estas cuestiones.

Y por último, la organización y gestión del museo se constituye como dificultador de la entrada de las TIC en los museos. En este sentido, la falta de tiempo y dedicación para incorporar las TIC en los programas educativos $(73,3 \%)$, los escasos y desactuali- 
zados recursos digitales con los que cuenta el museo $(71,1 \%)$, así como la falta de estrategias de formación tecnológica (60\%) son dificultades que una gran mayoría de los encuestados reconoce. Además, los recursos digitales disponibles se emplean mayoritariamente para la publicidad del museo y la gestión de las visitas $(62,3 \%)$.

\begin{tabular}{|l|c|c|c|}
\hline \multicolumn{1}{|c|}{ Ítem } & Media & DT & $\begin{array}{c}\text { \% de encuestados de } \\
\text { acuerdo y totalmente } \\
\text { de acuerdo }\end{array}$ \\
\hline $\begin{array}{l}\text { La falta de tiempo y dedicación dificultan la } \\
\text { incorporación de las TIC en los programas } \\
\text { educativos de los museo }\end{array}$ & 4,13 &, 919 & 73,3 \\
\hline $\begin{array}{l}\text { Los recursos digitales con los que se cuenta en } \\
\text { los museos no son suficientes y/o no están ac- } \\
\text { tualizados }\end{array}$ & 4,07 & 1,053 & 71,1 \\
\hline $\begin{array}{l}\text { Los recursos digitales disponibles se emplean } \\
\text { prioritariamente para la publicidad del museo y } \\
\text { gestión de las visita }\end{array}$ & 3,89 & 1,027 & 62,2 \\
\hline $\begin{array}{l}\text { La falta de estrategias de formación en TIC en } \\
\text { los museos dificultan la innovación }\end{array}$ & 3,84 & 1,065 & 60,0 \\
\hline $\begin{array}{l}\text { La falta de competencias digitales de los educa- } \\
\text { dores de los museos dificultan la innovación de } \\
\text { los programas educativos con TIC }\end{array}$ & 3,84 &, 903 & 64,5 \\
\hline $\begin{array}{l}\text { Los educadores de los museos respaldan la } \\
\text { innovación con TIC }\end{array}$ & 3,73 &, 863 & 50,1 \\
\hline $\begin{array}{l}\text { Los visitantes no tienen conocimientos ni habi- } \\
\text { lidades básicas en TIC }\end{array}$ & 2,96 &, 878 & 26,6 \\
\hline $\begin{array}{l}\text { Hay desconfianza hacia las TIC de los educado- } \\
\text { res de museos }\end{array}$ & 2,64 &, 933 & 24,4 \\
\hline $\begin{array}{l}\text { Los educadores de museos no ven el valor ins- } \\
\text { tructivo de las TIC en sus programas educativos }\end{array}$ & 2,64 & 1,048 & 19,0 \\
\hline $\begin{array}{l}\text { Los visitantes tienen una actitud negativa ante } \\
\text { el uso de las TIC en el museo }\end{array}$ & 2,76 &, 981 & 19,0 \\
\hline
\end{tabular}

Tabla 3. Media, desviación típica y porcentaje de encuestados de acuerdo y totalmente de acuerdo en la valoración de los ítems del cuestionario relacionados con las dificultades de integración y uso de las TIC en los museos.

\section{Conclusiones}

Una de las limitaciones tradicionales que se encuentran al valorar el potencial transformador de la tecnología en el aprendizaje escolar, es que su integración curricular se hace adaptándola a las metodologías existentes en las escuelas. La tecnología sirve para reforzar el modelo de escuela tradicional, incluso diferentes investigaciones han destacado que muchos de estos contextos escolares solo sufren una modernización conservadora al renovar infraestructuras tecnológicas, dejando inalteradas las constantes metodológicas. En otras publicaciones (Martínez Arbelaiz y Correa, 2009) hemos 
subrayado la importancia de la tecnología cuando se utiliza en contextos no formales, como los museos. Una de las fortalezas de estos espacios y experiencias educativas que se desarrollan en los museos es la posibilidad de diversificar los itinerarios de aprendizaje y favorecer otras experiencias que escapan a los limites curriculares de los programas escolares que se sufre en las escuelas. Los resultados obtenidos en esta investigación orientan una reflexión positiva, en el sentido que los museos por su temática, por la motivación de los visitantes y por la diversidad de los contextos ofrecen oportunidades de mejorar las experiencias de integración de las tecnologías.

Aunque los museos sufren las mismas presiones que el resto de las instituciones educativas y también las limitaciones que las creencias sobre el aprendizaje y la adquisición del conocimiento, sobre con qué y cómo se debe aprender. Los resultados que hemos presentado son en parte una garantía que va a permitir a los museos y sus educadores, realizar la doble tarea que tienen posibilidades de realizar. Por un lado, la difusión de los nuevos medios acercándolos al ciudadano; y, por otro lado desarrollando actividades de aprendizaje que integre los nuevos medios de otras manera a las que tradicionalmente se utilizan en el contexto escolar, pasando a asociarse a actividades de indagación, de colaboración y de representación creativa del conocimiento y de la experiencia. Una de las claves es aprovechar el elemento motivador de la visita al museo para acercar otro tipo de experiencias educativas asociadas con los nuevos medios a los visitantes.

Podríamos concluir que los educadores de museos, que han participado en este estudio, tienen una visión positiva de la tecnología. Reconocen la influencia en la motivación y las ventajas para la difusión y para la diversificación. Hemos hablado de ventajas, elementos importantes para la integración y uso eficiente de los nuevos medios digitales y de las dificultades de integración.

Este tipo de resultados son coherentes con la imagen que se tiene socialmente de la tecnología, tanto por las evidencias que se pueden constatar como por las ventajas asociadas mediáticamente. Un informe reciente sobre esta temática concreta señala (NMC, 2011) que en un periodo relativamente corto se prevé la incorporación de determinadas tecnologías emergentes. Entre estas se destacan en el informe los "mobile apps", "tablets", "realidad aumentada" y "smart objects".

Mientras siga habiendo demanda de los visitantes hacia estos nuevos medios y oportunidades de investigación y desarrollo en los museos y espacios de presentación del patrimonio, las tecnologías van a jugar un papel dinamizador del cambio en estas instituciones. Las actitudes y opiniones positivas de los educadores de museos (una gran mayoría sitúa en la necesidad de una actitud positiva hacia las TIC de todas las personas relacionadas con el museo), responden a un cambio en su perfil tradicional, donde la cultura digital se asume como parte importante de su identidad. Sin ser la solución a la mejora de la calidad de los programas educativos, los medios digitales pasan a constituirse como elementos fundamentales de la actividad didáctica formal o informal. Algo que parece no se puede cuestionar cuando hablamos de las misiones de los museos, que ya hoy no se pueden entender sin estos nuevos medios. 


\section{Referencias bibliográficas}

BALANSKAT, A., BLAMIRE, R. Y KEFALA, S. (2006). The ICT Impact Report. A review of studies of ICT impact on schools in Europe European Schoolnet. European Communities. Recuperado de

$\mathrm{http} / / /$ ec.europa.eu/education/doc/reports/doc/ictimpact.pdf.

BECTA. (2009). Harnessing technology review 2009. The role of technology in education and skills. Recuperado de http://dera.ioe.ac.uk/1422/1/becta_2009_htreview_report.pdf.

COLÁS, M. P. Y BUENDIA, L. (1994). Investigación Educativa. Sevilla: Alfar.

CONDIE, R. Y MUNRO, B. (2007). The impact of ICT in schools - a landscape review. Recuperado de http://dera.ioe.ac.uk/1627/1/becta_2007_landscapeimpactreview_report.pdf.

CORREA, J. M. Y IBAÑEZ, A. (2005). Museos, tecnología e innovación educativa: Aprendizaje de patrimonio y arqueología en territorio Menosca. REICE, 3(1), 880-894.

CORREA, J. M. (2008). Museos y tecnología: más que objetos y visitas virtuales. Comunicación y Pedagogía, 231-232, 48-52

CORREA, J. M. (2011). Antropología audiovisual y tecnología educativa: explorando nuevas formas de construir el conocimiento. En A. Garcia-Vera y H. Velasco (Coords), Antropología audiovisual: medios e investigación en educación (pp. 5367). Trotta: Madrid.

CORREA J. M. Y JIMENEZ DE ABERASTURI, E. (2011). Proyecto Museos Vivos: participación social y alfabetización digital. En M. Asensio y E. Pol (Coords). Lazos de luz azul. Museos y tecnología 1, 2 y 3.0 (pp. 255-272).

CORREA, J.M.; IBAÑEZ, A.; Y JIMENEZ DE ABERASTURI, E. (2006). Lurquest: Aplicación de tecnología m-learning al aprendizaje del patrimonio. Iber. Didactica de las Ciencias Sociales, 50, 109-123.

CORREA, J.M., LEON, M. Y FERNANDEZ, L. (2010). Definition of a didactic model for digital media literacy by means of a community narrative of heritage reconstruction and interpretation. Procedia - Social and Behavioral Sciences, 9, 831-834.

DRENT, M., Y MEELISSEN, M. (2008). Which factors obstruct or stimulate teacher educators to use ICT innovatively? Computers and Education, 51(1), 187-199.

ELY, D. P. (1999). Conditions That Facilitate the Implementation of Educational Technology Innovations. Educational Technology, 39(6), 23-27.

FALK, J. Y DIERKING, L. (2000). Learning from museums. Altamira Press: Walnut Creek.

HEIN, G. (1998). Learning in the museum. Routledge: Nueva York.

HOOPER-GREENHILL, E. (1999). The educational role of the museums. Routledge: Nueva York. 
IBAÑEZ A., VICENT, N., ASENSIO M. Y CORREA J. M. (2011). GPS+PDA, el uso de dispositivos móviles complejos. En M. Asensio y E. Pol (2011), Lazos de luz azul. Museos y tecnología 1, 2 y 3.0 (pp 175-192)

IBAÑEZ, A., ASENSIO, M. Y CORREA, J. M. (2011). Mobile learning y patrimonio: aprendiendo historia con mi móvil, mi GPS y mi PDA. En A. Ibañez (Ed.). Museos, redes sociales y tecnología 2.0 (pp 59-88). Universidad del País Vasco: Bilbao.

LEON, M. CORREA, J. M. ARAMBERRI, J. Y JIMENEZ DE ABERASTURI, E, (2010). Exploring ways to enhance permanent and collaborative learning by reusing online digital content. Procedia - Social and Behavioral Sciences, 9, 835-838

LOSADA, D., KARRERA, I. Y JIMENEZ DE ABERASTURI, E. (2012). Factors Facilitating Successful Educational Technology Innovations in the School. Revista de Psicodidáctica, 17(1), 243-264.

MARTINEZ ARBELAIZ, A.; CORREA, J. M.(2009). Can the Grammar of schooling be changed? Computers \& Education 53, 51-56.

MUMTAZ, S. (2000). Factors Affecting Teachers' Use of Information and Communications Technology: a review of the literature. Journal of Information Technology for Teacher Education, 9(3), 319 - 342

NMC (2011). Horizon Report Museum Edition 2011. Recuperado de http://www.nmc.org/publications/horizon-report-2011-museum-edition.

\section{Correspondencia con los autores}

José Miguel CORREA GOROSPE

E-mail: jm.correagorospe@ehu.es

Daniel LOSADA IGLESIAS

E-mail: daniel.losada@ehu.es

Estibaliz JIMENEZ DE ABERASTURI APRAIZ

E-mail: estitxu.aberasturi@ehu.es

Universidad del País Vasco - Euskal Herriko Unibertsitatea

Escuela Universitaria de Magisterio de San Sebastián

Plaza Oñati, 3

20018 - San Sebastián 